\title{
RFID Integrated Computer-Aided Laboratory Support Services (RFID ICLASS)
}

\author{
Joy V. Santelices and Benilda Eleonor V. Comendador
}

\begin{abstract}
The paper is about the integration of an online technical support request services, and monitoring of equipment, devices, \& peripherals using RFID in the computer laboratory rooms. The system includes tracking, adding, deleting, real time physical count, trace history, notifications on the technical support requests; and generating of reports. The purpose of this study is to develop and evaluate the use of RFID in an Integrated Computer- Aided Laboratory Support System in Universities and Colleges. The following results were derived: the level of assessment on the existing technical support services of the institution is very satisfactory; the level of acceptance of RFID-ICLASS in terms of functionality, reliability, usability, efficiency, portability and maintainability is highly acceptable; the level of assessment of the effects of RFID-ICLASS was very strong. Based from these results the use of RFID-ICLASS reflects transparency in record keeping and improves the service to the students of the universities.
\end{abstract}

Index Terms-Computer-aided, integration, laboratory support system, RFID.

\section{INTRODUCTION}

Information and Communication Technologies (ICT) are central to any economy or country today. These are removing boundaries in all disciplines, and for the last three decades there has been greater impetus given by Higher Education Institutions (HEIs) to set-aside larger budgets into the systematic development of technological infrastructures. A belief exists that HEIs (e.g. colleges, institutes, universities, etc.) have surpassed the ability to affect the successful transfer of skills and technological "know how" into the traditional classroom [1].

Technology in developing countries like the Philippines can be costly to access and construct. But despite such limitations, ICTs are considered integral to many initiatives in colleges and universities in the country and worldwide. As a popular view among the new generation including students and academics, scholars and futurists, the Internet or the Web has become "basic" rather than improvement innovation as well because of their huge societal impact [2]. The wide array of mobile phones with the ipod, iphone, cloud, and applications like itunes have revolutionized education and the

Manuscript received March 1, 2016; revised April 18, 2016; This work was supported in part by the Polytechnic University of the Philippines.

J. V. Santelices is with the Polytechnic University of the Philippines, Philippines. She is also with Catanduanes State University, Philippines (e-mail: yohjsv@yahoo.com).

B. E. V. Comendador is with the Polytechnic University of the Philippines, Philippines (e-mail: bennycomendador@yahoo.com). music industry, resulting to shifts in thinking and formulated questions that said innovations qualify as a series of basic innovations together with the internet. The argument for considering these and other innovations such as network enablers in Facebook, Linked-In, e-commerce, and the worldwide web as basic is reinforced as social media supported by wireless connectivity are now opening up an entirely new approach to individual entrepreneurial activities [3], [4]. The educative activities are not exempt from these innovations and with the technological innovation and long wave theories [2] reaching and transforming many Philippine HEIs with the smaller and larger universities, the need to be engaged in intriguing inquiries continue to redirect many graduate researchers. Technology support for school management such as Computer Laboratories Management (CLM) will be an interesting emphasis for a decision to address a myriad of gaps in maximizing IT Services in the complex university system.

CLM Services as described in this study emphasize IT services by defining online technical support request for laboratory users and monitoring/tracking of computer laboratory resources, hereto identified as Equipment, Devices and Peripherals (EDPs). The concepts of tracking [5] will require a wireless Automatic Identification and Data Capture (AIDC) technology, considered as "the next big thing" in the management and "the next revolution in supply chain" [6]. This is known as Radio-Frequency Identification (RFID), a common technology used in tracking items having enormous applications from business, to medicine and to military or crime science. RFID is the wireless non-contact use of radio-frequency electromagnetic fields to transfer data, for the purposes of automatically identifying and tracking tags attached to objects [7].

The effective use of technology in universities, businesses, and among individuals requires meticulous attention to the monitoring and maintenance of IT/ICT equipment. Successful monitoring of equipment needs a regular conduct of listing of EDPs. For sizable institutions including colleges/universities will need more time, manpower and paper design forms to conduct the monitoring and listing of EDPs with minimal errors. Difficulties in monitoring and documenting stolen and under maintenance/service PC units are also observed. Moreover, technical support service delivery is at a problematic state because of the limited technical support staff in the university. Considering that the members of the technical staff have other things to attend to an integrated system is most likely the most appropriate innovative way for inclusion in the computer laboratories of the university under consideration.

Technical staffs responsibilities are: 1). Provides technical 
support assistance to faculty, students and staff; 2). Makes recommendations to improve and purchase systems or equipment for accessibility, functionality and safety of facility and equipment; 3). Sets-up, installs, configures, maintains, replaces, and upgrades a variety of workstations both software and hardware; 4). Troubleshoots, repairs and analyzes software, hardware and network problems to ensure optimum performance; 5). Maintains inventory of computer laboratory and department workstations, hardware, software, licenses, materials and supplies.

RFID technology was chosen to effectively monitor the EDPs in the computer laboratories of the universities and possibly in the future, the whole university. RFID has been widely used to increase management efficiently by reducing time and effort and does not need to be directly placed under the reader not like the barcodes.

Equipment inventory/monitoring and technical support request in the existing system at Catanduanes State University, where the pilot test was done, are still done manually. Inventory/Monitoring of equipment is being conducted annually at CICT usually month of April. Technicians are the one who are responsible for the manual counting and checking of computer units and its components. Since it is physically counted by hand and by sight it is started having a printed empty Inventory Report form then they take a physical count and write the value or data needed on the form, after the physical counting of all the computers in every laboratory rooms, the technician prepare the inventory report to be submitted to the Dean of the College using MSExcel software. In technical support request, when malfunction of EDPs are detected, the laboratory users will fill-up the Technical Support Request Form (TSRF) and submit it to the Laboratory Assistant (LA) then the TSRF will be given to the technician. After checking the problem the technician will ask the Dean to verify the action taken on the problem encountered by the user and sign on the TSRF. The stated procedure requires human work and effort that is prone to human errors. Records of equipment were inaccurate because records might misplaced and not regularly updated. Technical support request were addressed not on time and not monitored.

With the high demand of work of a computer technical support team in the university, they must have a technological tool or an integrated support system that can help them in carrying out a number of tasks and functions without sacrificing the other responsibilities. Systems integration in IT is the process of linking together different computing systems and software applications physically or functionally, to act as a coordinated whole [8]. As such, the compelling needs to emphasize by designing and developing systems following the integrative approach that shows great promise by embedding the so-called RFID technology.

This study therefore puts premium of an integration of the different technological tool which is the monitoring of EDPs and online technical support to effectively, efficiently and appropriately improve the management of computer laboratories of the university and can also help to improve the teaching and learning process conducted in the laboratory rooms, if students and faculty concerns about technical support request is answered on the right time.

\section{THE DEVELOPED SYSTEM}

\section{A. System Architecture}

Fig. 1 shows the system architecture of the system. RFID ICLASS will be deployed in a computer laboratory rooms and in the technical room. The system can be accessed by the laboratory users in the computer laboratory rooms and the technical staff and System Administrator can access also the system in the technical room.

The programming language used in the development of the equipment monitoring module of the system to collect the information needed from RFID reader was VB.Net while the programming language used to develop the web-based online technical support module was PHP(Hypertext Preprocessor). Both modules store and retrieve data to and from the database using MySQL.

RFID tag is attached to the computer laboratory EDPs, and an RFID reader was used to record and verify the RFID tags in the area in each Laboratory. Students and faculty are encouraged to avail online technical support while they are inside the computer laboratory room. Data which are stored at the server can lead to no difficulty of the management in ascertaining the whereabouts of EDPs and record of the history information. Online monitoring by an authorized user or system administrator is also available for a sound and effective decision making of management.

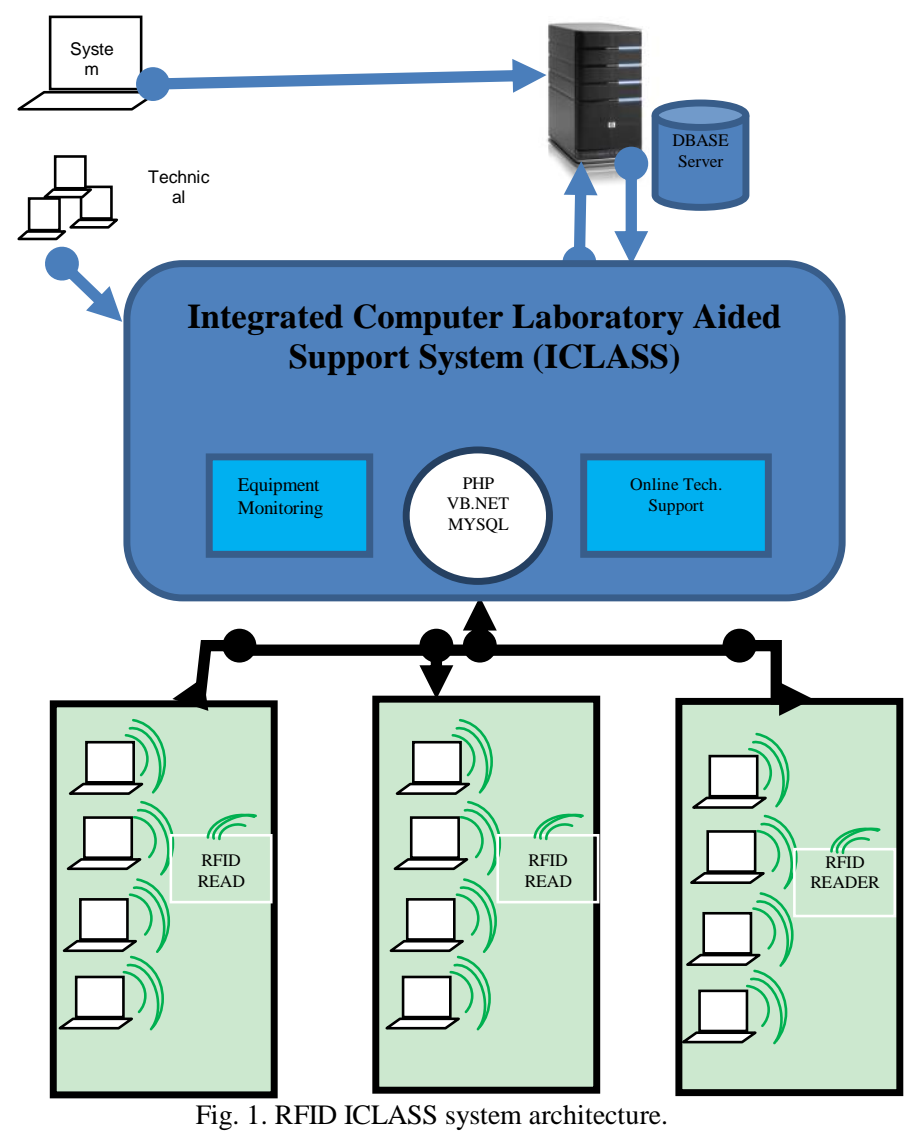

\section{B. System Features}

This system is a computer laboratory equipment, devices and peripherals EDPs monitoring, integrated with an online technical support for the different end-users (faculty, students, technical staff, and system administrator). 
The system developed for monitoring equipment in CSU includes 1) tracking of the equipment in the computer laboratory rooms; 2) adding and deleting of equipment, devices and peripherals (EDPs); 3) real time physical count of EDPs; 4) generate or trace EDPs history; and 5) generate reports on: a) serviceable and unserviceable units; and b) actual count or list of EDPs in the laboratory rooms.

The Online Technical Support is consists of 1) technical support request notification for the technicians; 2) Storage and retrieval of technical support request and reflect what solutions were made by the technician verified by the immediate supervisor; and 3) generation of reports reflecting the Job Requests and Acknowledgement reports by the technicians

The system was embedded with RFID technology which requires initial registration of users to access the ICLASS. Users of the system are classified into three levels that is -1) the administrator; 2) the technicians; and 3) laboratory users (faculty and students).

The system administrator of ICLASS is responsible for the registration of users, wherein the username is provided, for the student it is the Student Number ( $\mathrm{SN}$ ) and employee number was used for the technicians and faculty. The default password will be the inverse of their lastname. During the first login, users can change the default password. Both monitoring and technical support can be accessed by the administrator. Reports that can be generated from the system by the system administrator are the following: a. report on job requests; b. report on list of EDPs; c. reports on serviceable EDPs; d. reports on not serviceable EDPs.

Technicians are having the authority of: 1) Tracking/tracing of the EDPs; 2) Adding and deleting of EDPs; 3) Providing announcements for the laboratory users; 4) Viewing Technical support request notification from laboratory users; 5) Updating the technical support request made by the students; 6) Retrieving the technical support request verified by the immediate supervisor/end user; 7) Generate reports on equipment history and technician's accomplishment report.

The laboratory users can ask for technical support online by identifying the location of the computer laboratory room, Computer Number and the Computer Problem Description. They can also view updates on the requested technical support.

Since Passive RFIDs are used in this study, there is a maximum distance of the tagged equipment to the RFID reader so that it will be recognized. Only EDPs with RFID tags that are inside the computer laboratory rooms are considered. Software is not included in the monitoring system.

\section{RESEARCH MethodS AND TECHNIQUES}

The researcher used the developmental method of research to establish RFID-ICLASS which would be used by the faculty members, technical staff and students. Data were gathered through survey questionnaire which were distributed to the respondents using purposive sampling to the faculties and technicians; and systematic stratified random sampling to the students. Systematic stratified random sampling was done by placing all the names of the students in a box. The first three lots will be drawn, the fourth lot will be considered as the first respondents. This would be the procedure until all respondents are completed.

The figure is shown labeled as Fig. 2 is the conceptual framework of this study. It was consisted of three phases that shows the flow of the study leading to the development of the system. The first phase or inputs was concerned with the collection of management information process flow thus resulting to user's technical support system services delivery, technical support system resources utilization, materials/facilities setup, and support services problems and solutions that served as conceptual inputs. The $2^{\text {nd }}$ is the process phase which involved the development and design of the proposed system and the level of respondent's acceptability of the system being proposed in terms of security, accessibility, efficiency, and accuracy. The $3^{\text {rd }}$ is the final phase or outputs evaluated the acceptance of the proposed study and ensure the success of the proposed system. It also featured the implemented capabilities of the system.

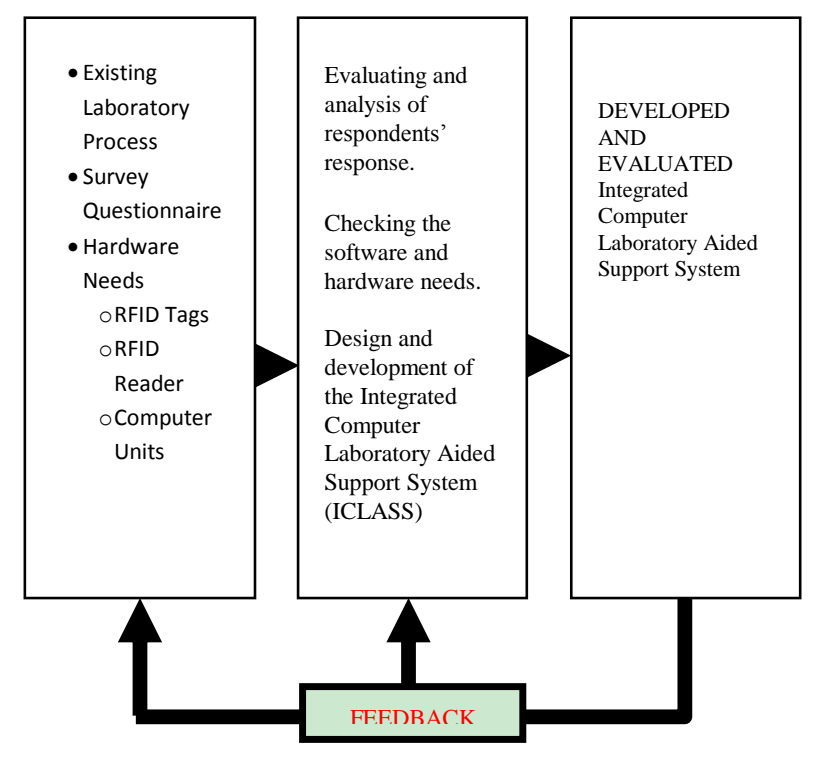

Fig. 2. RFID ICLASS conceptual framework.

The research instrument is consist of: 1) What is the level of assessment of the existing technical support services; 2) Level of acceptance of RFID-ICLASS in terms of functionality, reliability, usability, efficiency, maintainability and portability, and 3) The level of assessment on the effects of RFID-ICLASS to the institution.

To categorize the responses given by the respondents the four-point Likert Scale was used as shown in Table I.

TABLE I: FOUR-POINT LIKERT SCALE

\begin{tabular}{|c|c|l|}
\hline Scale & Quantitative Rating & \multicolumn{1}{|c|}{ Qualitative Rating } \\
\hline 4 & $3.25-4.00$ & (E) Excellent \\
\hline 3 & $2.50-3.24$ & (VS) Very Satisfactory \\
\hline 2 & $1.75-2.49$ & (S)Satisfactory \\
\hline 1 & $1.00-1.74$ & (NI) Needs Improvement \\
\hline
\end{tabular}

The Weighted mean was computed to determine the level of assessment of technical support services of the institution, the level of acceptance of RFID-ICLASS and level of assessment of the effects of the new system to the institution. Frequency count was used to determine the number of 
responses in each item.

Since the pilot tested was done at Catanduanes State University-College of Information and Communications Technology, the participants were eleven $(n=11)$ faculty members, four technical staff $(n=4)$, and 271 students which derived from the population in each level of laboratory user as shown in Table II and the total number of respondents is 286. For the students the sample size was determined using SLOVIN'S equation which is; $n=N /(1+N e 2 \neg)$, where $\mathrm{n}$ is the sample size, $\mathrm{N}$ the population of the study and e is the error margin.

TABLE II: RESPONDENTS OF THE STUDY

\begin{tabular}{|cl|c|c|}
\hline \multicolumn{2}{|c|}{ Strata } & Population, N & Sample size, $\mathbf{~}$ \\
\hline 1. & Students & 841 & 271 \\
\hline $2 . \quad$ Faculty members of CICT & 11 & 11 \\
\hline 3. & Technical staff & 4 & 4 \\
\hline Total & $\mathbf{8 5 6}$ & $\mathbf{2 8 6}$ \\
\hline
\end{tabular}

\section{RESULTS AND DISCUSSIONS}

This study was conducted to assess the existing computer laboratory technical support and the effects of using RFID-Integrated Computer Laboratory Aided Support System (ICLASS) in the institution. In addition, it is also sought to evaluate the level of acceptance on RFID -Integrated Computer Laboratory Aided Support System (ICLASS) in terms of: functionality; reliability; usability; efficiency; maintainability; and portability.

The findings revealed that 1). The level of assessment of the existing technical support services on the institution is 3 or very satisfactory; 2). The level of acceptance of RFID-ICLASS in terms of functionality is 3.59 or which is also highly acceptable; 3 ). The level of acceptance in terms of reliability is 3.59 or 4 or a descriptive rating of highly acceptable; 4). The level of acceptance in terms of usability is 3.49 or 4 or a descriptive rating of highly acceptable; 5). The level of acceptance in terms of efficiency is 3.48 or 4 or descriptive rating of highly acceptable; 6). The level of acceptance in terms of maintainability is 3.47 or 4 or descriptive rating of highly acceptable; 7). The level of acceptance of RFID-ICLASS in terms of portability is 3.5 or 4 or a descriptive rating of highly acceptable; and 8 . The level of assessment of the effects of RFID-ICLASS to the institution is 3.51 or 4 or descriptive rating of very strong.

\section{CONCLUSIONS AND FUTURE WORKS}

On the evaluation of the respondents, the following conclusions were derived: 1 . The level of assessment of the technical support services of the institution is very satisfactory but still needs improvement or enhancement; 2 . The level of acceptance of the RFID-ICLASS in terms of functionality, reliability, usability, efficiency, portability and maintainability is highly acceptable; 3 . The level of assessment of the effects of RFID-ICLASS to the institution is very strong, therefore, the developed system gives quality service to the institution.

Based on the conclusions of the study the following recommendations are offered: 1). There should be close monitoring of the equipment which are installed to notice if they are functional or if they are under repair, in order that the technical support services will attain a high degree of accuracy or "excellent"; 2). The use of RFID-ICLASS reflects transparency or honesty in record keeping and in providing useful data for research and reference, therefore its high acceptability in terms of functionality, reliability, usability, efficiency, maintainability and portability must be maintained; $3)$. Integration of other sub-system like users monitoring or attendance checking is also recommended.

\section{ACKNOWLEDGMENT}

J. V. Santelices, and B. E. V. Comendador thank all who encouraged, supported, and spend time to help for the success of this study.

\section{REFERENCES}

[1] D. Georgina and M. Olson, "Integration of technology in higher education: A review of faculty self-perceptions," Internet and Higher Education, vol. 11, 2008, pp. 1-8.

[2] H. A. Linstone and T. Devezas, "Technological innovation and the long wave theory," Technological Forecasting and Social Change, vol. 79, pp. 414-416, 2012.

[3] W. Isaacson, Steve Jobs, Simon \& Schuster, New York, 2011.

[4] J. Gertner, "Make or break," New York Times Magazine, Aug. 28, 2011, p. 45 .

[5] E. Valero et al., "Evolution of RFID applications in construction: A literature review," Sensors 2015, vol. 15, pp. 15988-16008, 2015.

[6] S. F. Wamba and K. Michael, An Information Systems Design Theory for an RFID.

[7] Wireless \& IP Technologies (WIT). (December 2014). Why RFID? [Online]. Available: http://www.wit-solutions.net/\#!rfid-/cq4k

[8] (August 20, 2013). System integration definition. [Online]. Available: http://en.wikipedia.org/wiki/

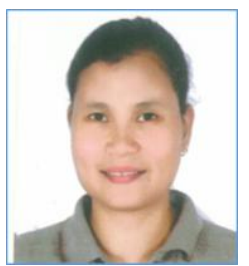

Joy V. Santelices is a candidate for master of science in information technology at Polytechnic University of the Philippines and currently employed at Catanduanes State University (CSU) College of Information and Communications Technology, Virac, Catanduanes, Philippines as Instructor I.

She is a member of IAMURE Multidisciplinary Research, Philippine Society of Information Technology Educators (PSITE) Foundation, Inc., Philippine Association of Researchers \& Statistical Software Users, Philippine Schools, Universities, Colleges, Computer Education and Systems Society (PSUCCESS).

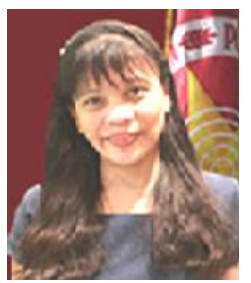

Benilda Eleonor V. Comendador was a grantee of the Japanese Grant Aid for Human Resource Development Scholarship (JDS) from April 2008 to September 2010. She obtained her master of science in global information telecommunication studies (MSGITS), major in project research at Waseda University, Tokyo Japan in 2010 and was commended for her exemplary performance in completing the said degree from JDS. She finished her master of science in information technology at Ateneo Information Technology Institute, Philippines in 2002.

Presently, she is the chief of the Open University Learning Management System (OU-LMS) and the program chair of the master of science in Information Technology (MSIT) of the graduate school of the Polytechnic University of the Philippines (PUP). She is an associate professor and was the former chairperson of the Department of Information Technology of the College of Computer Management and Information Technology of PUP. She attended various local and international computer related trainings and seminars. She was the country's representative to the project management course in 2005, which was sponsored by the Center for International Computerization Cooperation (CICC) in Tokyo, Japan together with other 9 representatives from various ASEAN countries. She presented several research papers in various international conferences.

Prof. Comendador is a member of Science and Engineering Institutes, Philippine Computer Society and Philippine Schools, Universities, and Colleges Computer Education and Systems Society (PSUCCESS). 